\title{
A Robotic Assisted System Using Deep Learning for Navigation and Reading IoT Based Home Automation for Visually Impaired
}

\author{
Jayashree $\mathrm{S}^{\mathrm{a}, 1}$, Jaswanthi $\mathrm{R}^{\mathrm{b}}$, Harshitha $\mathrm{S} \mathrm{V}^{\mathrm{c}}$ \\ ${ }^{a 1, b} U G$ Scholar, Dept of ECE, Panimalar Engineering College, Chennai, India \\ ${ }^{c}$ Associate Professor, Dept of ECE, Panimalar Engineering College, Chennai, India
}

\begin{abstract}
This paper presents a personal guidance system to help the visually impaired people to face the world independently; this is achieved by transferring visual world into the audio world by alerting them with a voice message. Navigation assistance is included with object detection, so object interference in the path of navigation the vision deprived person is alerted about the object in the path. The system is also implemented with an IOT based load control to help them with household objects; the load control can be operated with the help of Blynk Application which is made enable with voice assistant. In this project we have used an algorithm, real time object detection using deep learning. This model is trained with more images to recognize an object. OCR is implemented for the reading assistance module in this system.
\end{abstract}

Keywords. Assistive technology, blind people navigation, visually impaired, reading assistant, deep learning Raspberry pi 3processor

\section{Introduction}

The ability of a human to see is one of the fundamental needs. Eyesight is a quintessential requisite as it gives us a perception of the world around us. Life hits hard on the visionless people. Globally more than 2 billion people are suffering from vision impairment. The people with destitute of vision have a hard time in day-to-day life even with simple chores. In case of older adults' vision impairment can be a higher risk of falls and fractures. The visual world has to be transformed to an audio world for them. The major challenge blind person faces is navigation and reading text. The majorly used aids for visually impaired people are guide dogs and white cane. This proposed system reduces dependency of other people to the blind person and give major support in indoor navigation. This system aspires to assist visually impaired in reading by providing audio information of the textual verses.

$1_{\text {jayashree }}$, UG Scholar, Dept of ECE, Panimalar Engineering College, Chennai, India, E-mail: jayashrees22@gmail.com 
This proposed system has a Raspberry Pi-3 processor which uses deep neural network for image processing. Web camera is the input device utilized which is connected to the processor. A real time image is captured by the web camera and is sent to the Raspberry Pi-3 processor for further processing.

\section{Related work}

M. H. Mahmud, R. Saha and S. Islam,[6] has proposed a Smart Walking Stick[6], which usesa group of sensors positioned at approximate places to detect the object and indicated with An Light Emitting Diode indicator. The entire walking stick is controlled by a PIC microcontroller [6]. A. Mocanu, V. Sita, C. Avram, D. Radu and A. Aştilean, [1] has proposed a walking cane with Assistive Navigation Application for Blind People using Embedded System. This paper proposes a system which by all means is competent to provide assistance to the visually impaired to navigate independently [1]. O. Gamal, S. Thakkar and H. Roth, [2] has proposed an Intelligent Assistive System for Visually Impaired People for Outdoor Navigation [2]. In the present paper, we propose a compact device consisting of text to speech converter along with outdoor navigation system and home automation. It thereby strongly helps the visually impaired and empowers them.

\section{Proposed work}

\section{a. Text to speech conversion}

In this proposed work we have used Optical Character Recognition (OCR) algorithm and Google text to speech technique. Optical Character Recognition is a procedure that devises the operation of electronically reconstructing the graphic text images and handwritten entries in order to detect the underlying text by converting it into encoded data which within realm of possibility interpreted by a computer. The process is deployed by initially scanning the image which encompasses textual verses and scrutinizing of the image in order to single out the characters. Consequently, upon recognition the character is transformed to encoded text. Initially the scanned image consisting of text and graphic elements is remodelled into a bitmap (matrix of black and white). Further it is pre-processed so as to adjust the brightness and contrast thereby boosting the accuracy of the process. The image is now classified into various zones based on the areas of importance i.e., where the textual data exists, to launch the extraction procedure. Finally, the zone containing text and be further be divided into lines and thereby into characters. The obtained characters are now used by software to match by using various comparison and detection algorithms. The OCR engine deployed here is Tesseract which incorporates various wrappers. Pytesseract is the wrapper used. Pre- possessing is the vital stage of the procedure. The obtained characters further go through text to speech (TTS) conversion. GTTS (Google-text-tospeech) is a python library which is deployed for the conversion which can further be saved as audio files. It also supports assorted languages which act as a convenience to people of various ethnicities. 


\section{b. Autonomous Navigation and Object Detection}

The target of the object detection and navigation system is to distinguish various instances of objects from a given dataset and help the user navigate to provided destination accordingly based on the obstacle. Indifferently, a small class of objects are detected in the image, but a large number of desirable locations exist and the scales at which the expected outcome occurs, and is to be discovered. Each time an object is detected it is proclaimed with a structure of information. This information could be as basic as the placement of an object, a particular location and scale, or the object characterization in accord to the bounding box. In other scenarios, this information is more descriptive and houses the parameters of a linear or non-linear transformation. In the following proposed system, the background subtraction can be used by demonstrating the fixed web camera and producing a foreground mask. It compares the frame with the normal one containing background images or models which comprises the static area of the current scenario. The remaining area is compiled as the background part of images per se.

The background subtractions are consummated using the raspberry pi camera. The Image is captured by the web camera which is set-up on the raspberry pi module. The image is extracted from the camera and is sent to the raspberry pi module followed by the execution of the python code. In the code, the generated signals originate from the deployment of the module and further sent to the navigator. By the amalgamation of sensors and raspberry pi module, the object can be detected. Based on the object detection the navigator directs itself towards the required destination

\section{c. Home Automation}

Home automation is not an envisioned idea but is already persisting right from 2000's. In our project we have used Blynk application which is an advanced platform that grants you to promptly build an interface with a view to regulate and monitor an extensive range of hardware components from our gadgets without sophisticated code. Blynk application comprises of a unique identifier designated to individual user. On initiating the internet connection to the device i.e., the Raspberry Pi, the Blynk application functioning on the gadget establishes the connection to its server engaging the unique identifier established earlier. The server then indicates the application on the gadget, and then establishes the connection [13].

In order to execute the basal procedures (like turning GPIO pin - off or on) there is no necessity to do any coding. On the other hand, transmitting the information from the Pi back to the device requires a considerate amount of code [13]. To make it voice controlled we have enabled Google assistant to the Blynk app via IFTTT which act as an intermediate between the two application. 


\section{Block diagram}

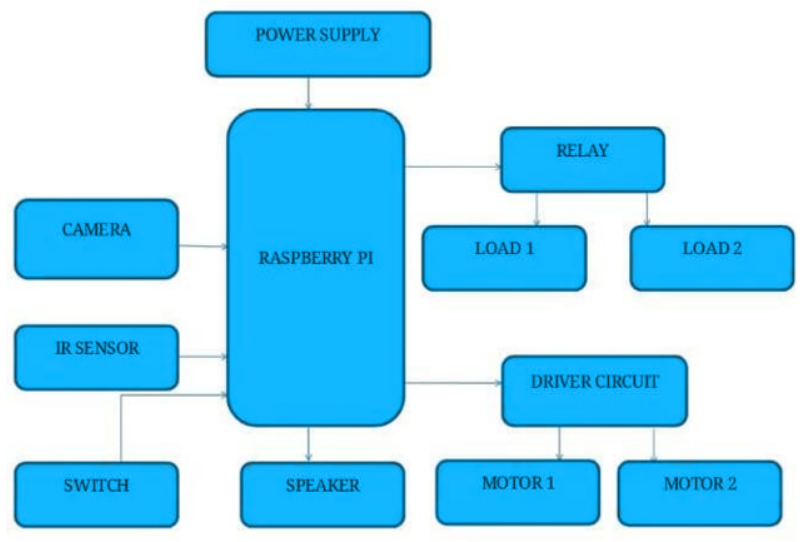

Figure 1. Diagram of proposed work

\section{Result and discussion}

Thus, the project we proposed tends to provide with a complete guidance to the visually deprived people. It may also be used as a helping aid robot for the elderly. In this system proposed here we used predefined or pre-stored path for indoor navigation, further GPS module can be established for outdoor navigation. The accuracy of the Reading assistant can be improved at a greater extent using higher end camera with higher resolution and by including high memory processor, graphics card like NVIDA which can even be developed to recognize more sophisticated scripts, cursive, handwriting texts etc.

\section{Conclusion}

The above proposed system is a simple architecture that transfigures text which is captured using a camera to audio data using Raspberry Pi module. It doesn't need any required skill or specific knowledge to operate it thus it reduces dependency to the blind. The navigation module assists the visually deprived in their way finding. It is also assisted with IOT based load control helps in ease of operating loads.

\section{References}

[1] Mocanu, V. Sita, C. Avram, D. Radu and A. Aştilean, Assistive Navigation Application for Blind People using a White Cane Embedded System, 2020 IEEE International Conference on Automation, Quality and Testing, Robotics (AQTR), Cluj- Napoca, Romania, 2020, pp.15,doi:10.1109/AQTR49680.2020.9129942.

[2] Gamal,S.ThakkarandH.Roth,TowardsIntelligentAssistiveSystemforVisuallyImpairedPeople:Outd oorNavigatinSystem,202024thInternationalConferenceonSystem Theory,ControlandComputing(IC 
STCC), Sinaia,Romania,2020,pp.390-397,doi:10.1109/ICSTCC50638.2020.9259682.

[3] Bourne,S.R.Flaxman,T.Braithwaite,M.V.Cicinelli,A.Das,J.B.Jonas,J.Keeffe,J.H.Kempen,J.Leashe r,H.Limburg,K.Naidoo,K.Pesudovs,S.Resnikoff,A.Silvester,G.A.Stevens,N.Tahhan,T.Y.Wongand H.R.Taylor,Magnitude,temporal trends, and projections of the global prevalence of blindness and distance and near vision impairment a systematic review and metaanalysis, TheLancetGlobalHealth,vol.5,no.9,pp. e888 - e897, September2017.

[4] PopulationReferenceBureau,2017WorldPopulationDatasheet, August 2017.

[5] A. T. Noman, M. A. Mahmud, H. Rashid and S. M. Saifur Rahman, Design and Implementation ofMicrocontrollerBasedAssistiveRobotforPersonwithBlindAutismandVisualImpairment,inInternat ional ConferenceofComputerandInformationTechnology(ICCIT), Chittagong,Bangladesh,2017.

[6] M.H.Mahmud,R.SahaandS.Islam,SmartWalkingStickAnElectronicApproachAssistVisuallyDisabl ed Person,InternationalJournalofScientific\&EngineeringResearch,vol.4,no.10,2013.

[7] Borenstein and I. Ulrich, The Guide Cane - A Computerized Travel Aid for the Active Guidance of Blind Pedestrians, in International Conference on Robotics and Automation, Albuquerque, New Mexico, 1997.

[8] R.A.ZeineldinandN.A.ElFishawy,FastandAccurateGroundPlaneDetectionfortheVisuallyImpairedf rom3DOrganizedPointClouds,inSAI ComputingConference,London,UK,2016.

[9] A.KaehlerandG.Bradski,LearningOpenCV3,Sebastopol,CA:O'ReillyMedia,Inc.,2016.

[10] J.Busck and H. Heiselberg, High Accuracy 3D Laser Radar, in Society of Photo-Optical Instrumentation Engineers, Florida, USA,2004.

[11] C.Stoll,R.PalluelGermain,V.Fristot,D.Pellerin,D.AlleyssonandC.Graff,NavigatingfromaDepthIma ge ConvertedintoSound,Hindawi,vol.2015,no.10.1155/2015/543492,p.9,2015.

[12] StereoLabs,How does the ZED work?-Help Center| Stereolabs,[Accessed8May2019].

[13] www.bc-robotics.com, - internet source. 